This is the accepted version of the article:

Magrasó A., Frontera C.. Comparison of the local and the average crystal structure of proton conducting lanthanum tungstate and the influence of molybdenum substitution. Dalton Transactions, (2016). 45. : 3791 - . 10.1039/c5dt04659a.

Available at: https://dx.doi.org/10.1039/c5dt04659a 


\title{
Comparison of the local and the average crystal structure of proton conducting lanthanum tungstate and the influence of molybdenum substitution
}

\author{
Anna Magrasó $^{\mathrm{a}, \mathrm{b}, \mathrm{c}^{*}}$, Carlos Frontera ${ }^{\mathrm{c}}$ \\ a Department of Chemistry, Centre for Materials Science and Nanotechnology (SMN), \\ University of Oslo, FERMiO, Gaustadalléen 21, NO-0349 Oslo, Norway \\ ${ }^{\mathrm{b}}$ Catalan Institute of Nanoscience and Nanotechnology (ICN2), CSIC and The Barcelona \\ Institute of Science and Technology, Campus UAB, Bellaterra, 08193 Barcelona, Spain \\ ${ }^{c}$ Institut de Ciència de Materials de Barcelona (ICMAB-CSIC), Campus UAB, E-08193 \\ Bellaterra, Spain \\ *Corresponding autor: annamagraso@gmail.com
}

\section{Keywords}

Lanthanum tungstate, proton conductor, structure, electrical conductivity, Mo-substitution

\begin{abstract}
We report on the comparison of the local and average structure reported recently for proton conducting lanthanum tungstate, of general formula to $\mathrm{La}_{28-\mathrm{x}} \mathrm{W}_{4+\mathrm{x}} \mathrm{O}_{54+\delta} \mathrm{V}_{2-\delta}$, and the impact of molybdenum-substitution to the crystal structure of the material. Partial replacement of $\mathrm{W}$ with 10 and 30 mol-\% Mo is investigated here, i.e. $\mathrm{La}_{27}\left(\mathrm{~W}_{1-\mathrm{x}} \mathrm{Mo}_{\mathrm{x}}\right)_{5} \mathrm{O}_{55.5}$ for $\mathrm{x}=0.1$ and 0.3 . This study allows the interpretation and the description of a disordered cation and anion sublattice in this material, which enables the understanding of the fundamental properties related to hydration, transport properties and degradation in lanthanum tungstate. The report shows that Mo-substituted lanthanum tungstate is a promising material as a dense oxide membrane for hydrogen separation at intermediate temperatures.
\end{abstract}




\section{Introduction}

Lanthanum tungstate (LWO) with a general formula corresponding to $\mathrm{La}_{28-\mathrm{x}} \mathrm{W}_{4+\mathrm{x}} \mathrm{O}_{54+\delta} \mathrm{V}_{2-\delta}$ is a proton conducting oxide by hydration of intrinsic oxygen vacancies at intermediate temperatures. ${ }^{1}$ LWO is a relatively pure proton conductor at low and intermediate temperatures and can therefore be used as electrolyte in proton-conducting solid oxide fuel cells. ${ }^{2,3,4,5}$ At higher temperatures, the material exhibits additional p-type and n-type electronic conductivity under oxidizing and reducing conditions, respectively. $2^{3,6}$ The material can, under reducing conditions, be used as a dense membrane for hydrogen separation due to the ambipolar transport of protons and electrons. $2^{7,8,}$ Hydrogen permeation in LWO is limited by electrons up to $\sim 1000{ }^{\circ} \mathrm{C}$. More recent materials' development have shown that partial replacement of $\mathrm{W}$ by Mo leads to increased electronic conductivity due to the higher reducibility of molybdenum compared to tungsten, with the advantange of not affecting protonic conductivity significantly.3,9,10 Therefore, Mo-LWO exhibits improved ambipolar transport and can be used as for hydrogen separation.

LWO exhibits a fluorite-type crystal structure $^{11}$ with the special feature that tungsten dissolves in lanthanum sites to form a stable electrolyte.1 A computational model was obtained from density functional theory (DFT) calculations to describe its real crystal structure for the first time.1 The model was later verified from a pair distribution function (PDF) study using both time-of-flight neutron and synchrotron data collected at room

temperature. ${ }^{12}$ Another study has reported an average model based on a combination of neutron and synchrotron data, and a local model based on PDF and EXAFS. ${ }^{13}$ The crystal structure of the Mo-substituted material has, to the best of our knowledge, not been reported yet.

The goal of the present study is first to compare the structural model for unsubstituted LWO extracted from DFT calculations reported by Magrasó and co-workers $1^{12}$ with the average model reported by Scherb et. al. ${ }^{13}$ Then, the crystal structure of Mo-substituted LWO $\mathrm{La}_{27}\left(\mathrm{~W}_{1-\mathrm{x}} \mathrm{Mo}_{\mathrm{x}}\right)_{5} \mathrm{O}_{55.5}$ (with $\mathrm{x}=0.1$ and 0.3 ) will be described based on newly acquired neutron and synchrotron diffraction data. Finally, the electrical properties of the materials will be reported. 


\section{Experimental}

Nanometric powders of $10 \%$ Mo-LWO $\left(\mathrm{Mo}_{0.1}\right.$; i.e. $\left.\mathrm{La}_{27} \mathrm{~W}_{4.5} \mathrm{Mo}_{0.5} \mathrm{O}_{55.5}\right)$ and $30 \%$ Mo-LWO $\left(\mathrm{Mo}_{0.3}\right.$, i.e. $\left.\mathrm{La}_{27} \mathrm{~W}_{3.5} \mathrm{Mo}_{1.5} \mathrm{O}_{55.5}\right)$ were synthesized by the EDTA combustion method, as described elsewhere. 3 The powders were fired at $500-1000{ }^{\circ} \mathrm{C}$ until the organics were removed, and thereafter ground and re-calcined at $1400{ }^{\circ} \mathrm{C}$ for 5 hours in order to obtain single phase with high crystallinity.

Neutron powder diffraction data for $\mathrm{Mo}_{0.1}$ and $\mathrm{Mo}_{0.3}$ were collected at $\mathrm{D} 2 \mathrm{~B}$ beamline of Institut Laue Langevin (Grenoble, France) in high flux mode using $\lambda=1.594 \AA$, samples were finely ground and enclosed at a cylindrical V sample holder. Synchrotron data were collected at MSPD (BL06, Alba synchrotron, Cerdanyola del Vallès, Spain, ${ }^{14}$ ) using multianalyzer detector (MAD). Using the single cut doble Si-111 monochromator, a short wavelength of $\lambda=0.4247 \AA$ was selected in order to reduce the absorbance the tungstate. The fine powder was embedded in a $3 \mathrm{~mm}$ in diameter capillary that was rotated during data collection. Data were refined by Rietveld method ${ }^{15}$ using FullProf program ${ }^{16}$. For comparison purposes, synchrotron data of the undoped sample $\left(\mathrm{Mo}_{0}\right)$ were collected at ID31 beamline of European Synchrotron Radiation Facility (Grenoble, France) with $\lambda=0.3999 \AA$.

Pellets of $\sim 20 \mathrm{~mm}$ diameter and $\sim 1 \mathrm{~mm}$ thickness were prepared for electrical measurements. Pt electrodes were painted on each side of the specimens and fired at $1000{ }^{\circ} \mathrm{C}$ for $30 \mathrm{~min}$. The conductivity was measured using an Alpha A impedance spectrometer + POTGAL interface (Novocontrol technologies) in a ProboStat ${ }^{\mathrm{TM}}$ measurement cell (NorECs, Norway) by the 2point 4-wire method. Impedance spectra were recorded in the $1 \mathrm{MHz}$ to $0.1 \mathrm{~Hz}$ frequency range with an oscillation voltage of $50 \mathrm{mV}$ in wet $\mathrm{H}_{2}$. The variation of conductivity with $\mathrm{H}_{2} \mathrm{O}$ was done in oxygen at constant water vapour partial pressure by mixing $\mathrm{O} 2$ bubbling through a $\mathrm{KBr}$ saturated water solution $\left(p \mathrm{H}_{2} \mathrm{O} \sim 2.5 \%\right)$ or through a $\mathrm{P}_{2} \mathrm{O}_{5}$ powder drying stage $\left(p \mathrm{H}_{2} \mathrm{O} \sim 3 \cdot 10^{-5} \mathrm{~atm}\right)$.

\section{Results and discussion}

\subsection{Disordered average structure}


There are two main (average) structural reports of LWO: the first structural model was reported by Magrasó et al. in $2009^{11}$ (and successive improvements of the first by incorporating computer modelling $1^{12}$ ), and a later work by Scherb et al in $2013,{ }^{13}$ which proposes a disordered structure for lanthanum tungstate. The two reported structures are similar but use different space groups (the first: $F \overline{4} 3 m$, the latter : $F m \overline{3} m$ ). Magraso et $\mathrm{al}^{11}$ reported the initial structural model with atoms sitting in regular positions according to the fluorite-type structure. Scherb et al. ${ }^{13}$ introduced the disorder by displacing the positions of one lanthanum and one oxygen slightly away from one of the symmetry elements of their Wyckoff positions while lowering their occupancies accordingly. More precisely, in the average structure one of the $\mathrm{La}$ is placed at the $24 d\left(0^{1 / 4} \frac{1 / 4}{4}\right)$ Wyckoff position but in the disordered structure it is moved away from one of the three mirror planes, along the binary axis and occupies Wyckoff position $48 h(0 y y)$ with half occupancy (and $y \sim 1 / 4)$. This leads to two very near "half" lanthanum ions. In a similar way, one of the oxygen anions is moved from the $32 f(x x x)$ to $96 k(x x z)$ by leaving the ternary axis along the mirror plane, giving rise to three oxygen anions with one third of the occupancy in the average structure.

We have tested this model of the "disordered" LWO description against the data used in ref. [1]. The details of the structure found by the joint refinement of SXRPD and NPD data are printed in Table 1 along with the agreement parameters. For the sake of comparison, we have also used the $F \overline{4} 3 m$ description to fit the same data (see supplementary information). As a result, we first conclude that the disorder description of the structure within $F m \overline{3} m$ SG $\left(\chi^{2}=3.62, \mathrm{R}_{\mathrm{B}}=3.36 \%\right.$ and $3.64 \%$ for SPXRD and NPD, respectively), c.f. Table 1 , is more accurate than the average description using $F \overline{4} 3 m$ without the disorder $\left(\chi^{2}=5.95\right.$ and $\mathrm{R}_{\mathrm{B}}=5.89 \%$ and $5.97 \%$ for SXRPD and NPD, respectively). For the refinements, we have fixed the $\mathrm{La} / \mathrm{W}$ ratio to be $27 / 5$ (=5.4) by substituting one of the $24 \mathrm{La}$ of the $48 \mathrm{~h}$ position by one $\mathrm{W}$. Oxygen content has been refined and the result of the stoichiometry is 31.4 oxygen ions at $32 \mathrm{f}$ position $(\mathrm{O} 2$, around $\mathrm{La} 1)$ and 24.0 at $96 \mathrm{k}$ position (around $\mathrm{W} 1)$, thus rendering an average six-fold coordination for W1. The total refined oxygen occupancy is, therefore, 55.4, while the theoretical content is 55.5 according to the formula $\mathrm{La}_{28-\mathrm{x}} \mathrm{W}_{4+\mathrm{x}} \mathrm{O}_{54+\delta} \mathrm{V}_{2-\delta}$ $(\delta=3 \mathrm{x} / 2)$ for $\mathrm{x}=1$.

\subsection{Disorder description vs. modelled local structure}


In agreement with the average model, the theoretical DFT calculations of the structure $1^{, 12}$ establish that tungsten is not cubically but octahedrally coordinated, and the $\mathrm{WO}_{6}$ octahedra adopt alternating directions. This confers an average $\mathrm{W}-\mathrm{O}$ coordination of 8 and a cube-like shape, although the maximum oxygens around tungsten is 6. La1 is mainly 8-fold coordinated forming relatively symmetric cubes, while La2 is coordinated with 6 or 7 oxygens in heavily distorted cubes. As mentioned earlier, the LWO structure is only stable when some tungsten dissolves in lanthanum sites $\left(\mathrm{La}_{28-\mathrm{x}} \mathrm{W}_{4+\mathrm{x}} \mathrm{O}_{54+\delta}\right.$, and it is now well established that the optimal composition is for $x^{\sim} 1$ ( $\mathrm{LWO} 4$, with $\mathrm{La} / \mathrm{W}$ ratio $\left.27 / 5=5.4\right)$. The "additional" tungsten (x) dissolves into La2 sites (not in La1), as this position has a highly distorted environment and is sufficiently flexible to the substitution by W. Lal does not accommodate $\mathrm{W}$ due to its more regular and stiffer environment and longer La-O bond length ${ }^{12}$. This theoretical structure has been extensively validated by several methods and has been the key to understand the functional properties of LWO, including hydration thermodynamics, electrical properties, and degradation. ${ }^{17}$ It is of major relevance that the DFT model fits very well with the experimental local structure determined by PDF. ${ }^{12}$

We have compared the disordered average structure found by diffraction with the local structure found by DFT-PDF by Kalland et al. ${ }^{12}$ The objective of this comparison is twofold. On the one hand, we aim to examine the compatibility between these two descriptions. On the other hand, we intend to understand the instant local environments in the disordered structure. Note that due to the fact that DFT can not model partial occupancies, the comparison is made using a $2 \times 1 \times 1 \mathrm{La}_{27} \mathrm{~W}_{5} \mathrm{O}_{55.5} \mathrm{~V}_{0.5}$ cell, i.e. $\mathrm{La}_{54} \mathrm{~W}_{10} \mathrm{O}_{111} \mathrm{v}_{1}$. For simplicity, we will refer to the structures as "disordered" and "DFT" structures. The procedure has been the following. First, we have generated all the atoms in the "disordered" cell, and compared their positions with those in the "DFT" structure. We have found, for every atom in the later, the closest atom of the same element in the former (except for the two $\mathrm{W}$ placed at La positions that were introduced in the DFT structure). The distance between the two atoms ("DFT" structure vs. the closest in the "disordered" structure), can be understood as a measure of the compatibility between the two. As a result, the mean distance is $0.13 \AA$ for the cations, and $0.19 \AA$ for all the anions. The reason for a larger disagreement of the anion sublattice is due to the oxygens that surround the "special" W that sits on La2 sites, which necessarily leads to shorter cation-oxygen distances for W-O than for La-O. This local arrangement (1 out of 24 positions) is poorly described in the "disordered" average structure. When excluding these 
oxygen ions, the average difference between the anions of the two structures reduces to 0.16 $\AA$. The list of ions is given as supplementary material. In addition to the substitution of two $\mathrm{W}$ atoms in La2 sublattice and one oxygen vacancy in the local structure, one must take into account that DFT calculations are made at zero kelvin temperature, while diffraction data are collected at RT. All these considerations make us to conclude that the disordered structure obtained by diffraction is fully compatible with the local structure found by DFT.

The overall comparison renders that the apparent eight-fold coordination of W1 (with partial anion occupancy) originally found with the $F \overline{4} 3 \mathrm{~m}$ SG indeed represents disordered octahedra in the local structure, as depicted in Figure 1 (passing through the disordered structure). From this picture, two corners (placed at opposite positions along a cube diagonal) of the original cube are always empty (in a dynamical way, illustrated as the different orientations of the diagonal containing the "vacancies" giving rise to the octahedra). The other 6 oxygen ions occupy the position (out of the three ones generated in the disordered structure) closest to the empty sites. This means that the theoretical oxygen content of the real cell $\left(\mathrm{La}_{27} \mathrm{~W}_{5} \mathrm{O}_{55.5} \mathrm{~V}_{0.5}\right)$ is much lower than that predicted from the fluorite structure $\left(\mathrm{La}_{27} \mathrm{~W}_{5} \mathrm{O}_{55.5} \mathrm{~V}_{8.5}\right)$. This indubitably has consequences when determining the hydration thermodynamics of $\mathrm{LWO}^{18}$, which was initially believed to hydrate very little from the theoretical oxygen vacancy content. It is now well established that the vacancy content corresponds to $\mathrm{La}_{28-\mathrm{x}} \mathrm{W}_{4+\mathrm{x}} \mathrm{O}_{54+\delta} \mathrm{V}_{2-\delta}$ (for a given stoichiometry, $\delta=3 \mathrm{x} / 2$ ). This additionally makes that the coordination of La2/W2 cations to decrease from eight to seven (in most of the cases), as depicted in Figure 2. This introduces a large distortion and asymmetry in La2/W2 environment that forces this cation to be displaced from $24 d\left(\begin{array}{l}1 / 1 / 4 \\ 1 / 4\end{array}\right)$ to $48 h\left(\begin{array}{lll}0 & y & y\end{array}\right)$ Wyckoff position. From the two positions generated, comparison with the local DFT structure renders that $\mathrm{La}$ moves towards the largest coordination thus introducing a correlation (at a short extent) that relates the orientation of the $\mathrm{WO}_{6}$ octahedra linked by the intermediate La2/W2 This local arrangement has been described using $P a \overline{3}$ SG by Scherb et al. ${ }^{13}$. Another interesting result of the comparison between the two structures is the possible role of W2 in the disordered structure. According to DFT calculations, these anions have a 6-fold coordination instead of the 7-fold of La2. This breaks the correlation depicted in Figure 2 between W1O6 octahedra that are connected through a W2 ion. 
This local description also allows the detailed study of the environment of oxygen anions. O2 are all connected to one La1 and to three La2/W2 positions (bond distances are printed in Table 1). According to bond valence sum (BVS) calculations, their charge would be -2.003 (-2 within the errors). Besides, O1 is bonded to W1 and also to three La2/W2 but BVS calculation render a charge of -1.89 , slightly below -2 . This result is for the average situation. In the local disordered structure one must expect that when the true local bond is towards a $\mathrm{W}$ ion, the charge of the $\mathrm{O}$ would be much lower than this. This could indicate that these oxygen ions are more susceptible to accept $\mathrm{H}$ atoms.

\subsection{Disorder description of Mo-doped system}

We have used the "disordered" structural description to obtain the joint refinement for Mosubstituted LWO. The refined patterns are plotted in Figures $3\left(\mathrm{Mo}_{0.1}\right)$ and $4\left(\mathrm{Mo}_{0.3}\right)$. The obtained atomic positions, bond distances (interpreted according to the "DFT" structure) and agreement parameters, are printed in Table 2. In both cases, we have found that Mo atoms replace $\mathrm{W}$ with no clear preference between $\mathrm{W} 1$ and $\mathrm{W} 2$ positions. The introduction of Mo implies an enlargement of cell parameter, in accordance with Amsif et al.9 This is in contradiction with size considerations, since $\mathrm{Mo}^{6+}(0.59 \AA)$ is smaller than $\mathrm{W}^{6+}(0.60 \AA) .{ }^{19}$ Thus, the enlargement of the unit cell volume could indicate reduction of some species due to the creation of additional oxygen vacancies, since molybdenum is more reducible than tungsten. Examining the values of the occupancies in the $\mathrm{O} 2$ site (where the oxygen vacancies are located) no significant difference can be found between undoped and Mo-10\% sample. For Mo-30\%, the oxygen occupancy is somewhat smaller, indicating an increase of oxygen vacancy concentration, supporting the partial reduction of $\mathrm{Mo}$ in $\mathrm{Mo}_{0.3}$. However, the associated error bar prevents us to asses this fact categorically. Looking at the bond distances, only La-O2 increases slightly in the undoped structure compared to the Mo-substituted LWO. Examining the local structure of both compounds, in a similar fashion as for the undoped case (bond distances are reported in the tables), we find that the estimated valence of $\mathrm{O} 1$ is -1.90 (for both Mo-10\% and Mo-30\%). This is below the expected value of -2 , and very similar to the value found for the undoped case.

\subsection{Electrical conductivity at intermediate temperatures}


The variation of the conductivity with water vapour partial pressure in $\mathrm{O}_{2}$ (at $500^{\circ} \mathrm{C}$ ) and the variation with temperature in wet $\mathrm{H}_{2}$ for LWO54 and the Mo-doped compositions are shown in Figures 5 and 6. These materials are mixed ionic-electronic conductors depending on the temperature and atmosphere $3^{, 9}$. Under oxidizing conditions, the conductivity of the materials increases largely with increasing water partial pressure. This is in accordance with the formation of conducting protons following the exothermic nature of the hydration reaction:

$$
v_{O}^{\bullet}+\mathrm{O}_{O}^{x}+\mathrm{H}_{2} \mathrm{O}=2 O H_{O}^{\bullet}
$$

The differences between the undoped and Mo0.3 are minor, which supports that the Mo substitution does not affect protonic conductivity much in this family of materials. There is, however, a small electron p-type contribution in $\mathrm{O}_{2}$, according to refs. ${ }^{3,9}$ In reducing conditions, the materials are mixed ionic-electronic conductors, and the electronic conductivity increases with increasing the Mo-content, as observed from Fig. 6. The increase in electronic conductivity is small for $\mathrm{x}=0.1$, while it is significant for $\mathrm{x}=0.3$. The fact that the electronic conductivity increases at higher Mo contents clearly indicates that additional electronic charge carriers are being formed, which is correlated, again, with the higher reducibility of Mo compared to $\mathrm{W}$. This in an effect that is strongly reflected under reducing conditions (c.f. Fig. 5), but it does correlate quite well with the assumption that additional vacancies are formed in Mo0.3 compared to Mo0.1 and LWO, even in oxidizing conditions (the samples were prepared in air).

It is worth to mention that LWO and Mo-doped LWO are stable materials towards $\mathrm{CO}_{2} .8$ Also, it was earlier concluded that Mo-doping does not seem to affect proton conductivity significantly (within the uncertainty of the extraction of the transport numbers using the EMF method). 3 This means that $\mathrm{Mo}_{0.3}$ exhibits increased ambipolar conductivity compared to LWO, and improved chemical stability compared to state-of-the-art membrane materials (such as $\left.\mathrm{SrCeO}_{3}\right)^{20,21}$. The variation of the ambipolar conductivity for various compositions is shown in Figure 7, which clearly shows that the ambipolar conductivity increases significantly with molybdenum concentration. Mo-substituted LWO is, therefore, proposed as the next generation materials for improved $\mathrm{H}_{2}$ separation in dense ceramic membranes. Several research groups are testing hydrogen separation mostly in bulk form ${ }^{10,22}$, and more 
advanced architectures such as hollow fibers ${ }^{23}$ or thin films ${ }^{24,25}$ with modified surface for increased surface kinetics are expected to come in the near future.

\section{Conclusions}

In summary, we have investigated the structural and transport properties of Mo-substituted $\mathrm{La}_{28-\mathrm{x}}\left(\mathrm{W}_{1-\mathrm{y}} \mathrm{Mo}_{\mathrm{y}}\right)_{4+\mathrm{x}} \mathrm{O}_{54+\delta} \mathrm{V}_{2-\delta}$ (with $\mathrm{x}=1$ and $\mathrm{y}=0.1$ and 0.3 ) and revisited the structure of the undoped case $(x=1, y=0)$. First, we have found that the disordered model proposed by Scherb et al. in Ref. [13] reproduces diffraction data (neutron and synchrotron x-ray powder diffraction) very satisfactorily. In addition, we have examined the compatibility of this disordered model with the local structure found by DFT calculations and found a quite good agreement. From this comparison, we conclude that $\mathrm{W}$ is, both dynamically and locally, in an octahedral environment, and that these octahedra are oriented in alternating directions. We also confirm that the structure can be locally described in the $P a \overline{3}$ space group and that the BVS calculations in this local environment indicate that $\mathrm{O} 1$, surrounding the positions shared by $\mathrm{La}$ and $\mathrm{W}$, present an average valence of around -1.9. This value is expected to be significantly lower (in absolute value) for those oxygen anions surrounding $\mathrm{W}$ ions. We interpret this as an indication that proton transport may take place through these oxygen ions (those bonded to a $\mathrm{W}$ ion).

Rietveld refinements of neutron and synchrotron powder diffraction data of Mo-substituted compounds also confirm the disordered structure is preserved for up to $30 \%$ Mo substitution. In these cases, the valence of $\mathrm{O} 1$ positions does not change with Mo substitution and, accordingly, the proton conductivity does not vary along the studied series.

In accordance with the conducting protons formation thanks to the exothermic hydration reaction, the conductivity of doped and undoped LWO largely increases with water partial pressure under oxidizing conditions. The high ambipolar conductivity and high stability towards acidic gases, makes Mo-substituted LWO a potential material for substituting current unstable state-of-the-art materials for dense ceramic hydrogen permeation membranes.

\section{Acknowledgements}

ILL is acknowledged for the provision of beamtime through EASY program (EASY217 and 218) and Dr. E. Suard for data collection. We thank European Synchrotron Radiation Facility 
for the provision of beamtime. We acknowledge ALBA synchrotron for the beamtime provided through proposal no. 2012010161 and Dr. F. Fauth by his help during data collection. We thank ESRF for the provision of beamtime and Dr. I. Margiolaki for her assistance during data collection. AM wishes to thank continuous funding from the Research Council of Norway $(\mathrm{RCN})$.

\section{References}

${ }^{1}$ A. Magrasó, J. M. Polfus, C. Frontera, J. Canales-Vazquez, L.-E. Kalland, C. H. Hervoches, S. Erdal, R. Hancke, M. S. Islam, T. Norby and R. Haugsrud, J. Mater. Chem., 2012, 22(5), $1762-1764$

${ }^{2}$ R. Haugsrud, Solid State Ionics, 2007, 178(7), 555-560

${ }^{3}$ A. Magrasó, J. Power Sources, 2013, 240, 583-588

${ }^{4}$ M.J. Zayas-Rey, L. dos Santos-Gómez, J.M. Porras-Vázquez, E. R. Losilla, D. MarreroLópez, J. Power Sources, 2015, 294, 483-493

${ }^{5}$ E. Quarez, Y. Oumellal, O. Joubert, Fuel Cells 2013, 13, 34-41.

${ }^{6}$ T. Shimura, S. Fujimoto and H. Iwahara, Solid State Ionics, 2001, 143, 117-123.

${ }^{7}$ S. Erdal, L.-E. Kalland, R. Hancke, J. Polfus, R. Haugsrud,T. Norby and A. Magraso, Int. J. Hydrogen Energy, 2012, 37(9), 8051-8055.

8 S. Escolastico, J. Seeger, S. Roitsch, M. Ivanova, W.A. Meulenberg, J. Serra, ChemSusChem 2013, 6, 1523-1532.

${ }^{9}$ M. Amsif, A. Magrasó, D. Marrero-López, J. C. Ruiz-Morales, J. Canales-Vázquez and P. Núñnez, Chem. Mater., 2012, 24(20), 3868-3877.

${ }^{10}$ E. Vøllestad, C. K. Vigen, A. Magraso and R. Haugsrud, J. Membr. Sci., 2014, 461, 81-88.

11 A. Magrasó, C. Frontera, D. Marrero-López and P. Núñez, Dalton Trans., 2009, $10273-$ 10283

${ }^{12}$ L.-E. Kalland, A. Magrasó, A. Mancini, C. Tealdi and L. Malavasi, Chem. Mater., 2013, 25(11), 2378-2384

${ }^{13}$ T. Scherb, S. A. J. Kimber, C. Stephan, P. F. Henry, G. Schumacher, J. Just, S. Escolastico, J. M. Serra, J. Seeger, A. H. Hill and J. Banhart, arXiv:1305.3385v1, 2013

${ }^{14}$ F. Fauth, I. Peral, C. Popescu, M. Knapp, Powder Diffraction, 2013, 28(S2), S360-S370.

${ }^{15}$ H. M. Rietveld, J. Appl. Crystallogr. 1969, 2, 65-71.

${ }_{16}^{16}$ J. Rodríguez-Carvajal, Phys. B Condens. Matter 1993, 192, 55-69.

${ }^{17}$ A. Magrasó, R. Haugsrud, Journal of Materials Chemistry A 2014,2, 12630-12641.

${ }^{18}$ R. Hancke, A. Magrasó, T. Norby and R. Haugsrud, Solid State Ionics, 2013, 231, 25-29.

${ }^{19}$ R. D. Shannon, Acta Crystallographica. 1976, A32, 751-767.

${ }^{20}$ T. Oh, H. Yoon and E. D. Wachsman, Solid State Ionics, 2009, 180, 1233-1239

${ }^{21}$ S. Hamakawa, L. Li, A. Li and E. Iglesia, Solid State Ionics, 2002, 148, 71-81

22 J.M. Polfus, W. Xing, M.-L. Fontaine, C. Denonville, P.P. Henriksen, R. Bredesen, J. Mem. Sci. 2015, 479, 39-45.

${ }^{23}$ Y. Chen, Q. Liao, Z. Li, H. Wang, Y. Wei, A. Feldhoff, J. Caro, AIChE Journal 2015, 61, 1997-2007.

${ }^{24}$ V. Gil, J. Gurauskis, C. Kjølseth, K. Wiik and M.-A. Einarsrud, Int. J. Hydrogen Energy, 2013, 38(7), 3087-3091. 
${ }^{25}$ W. Deibert, M.E. Ivanova, W.A. Meulenberg, R. Vaßen, O. Guillon, J. Memb. Sci. 2015, 492, 439-451. 


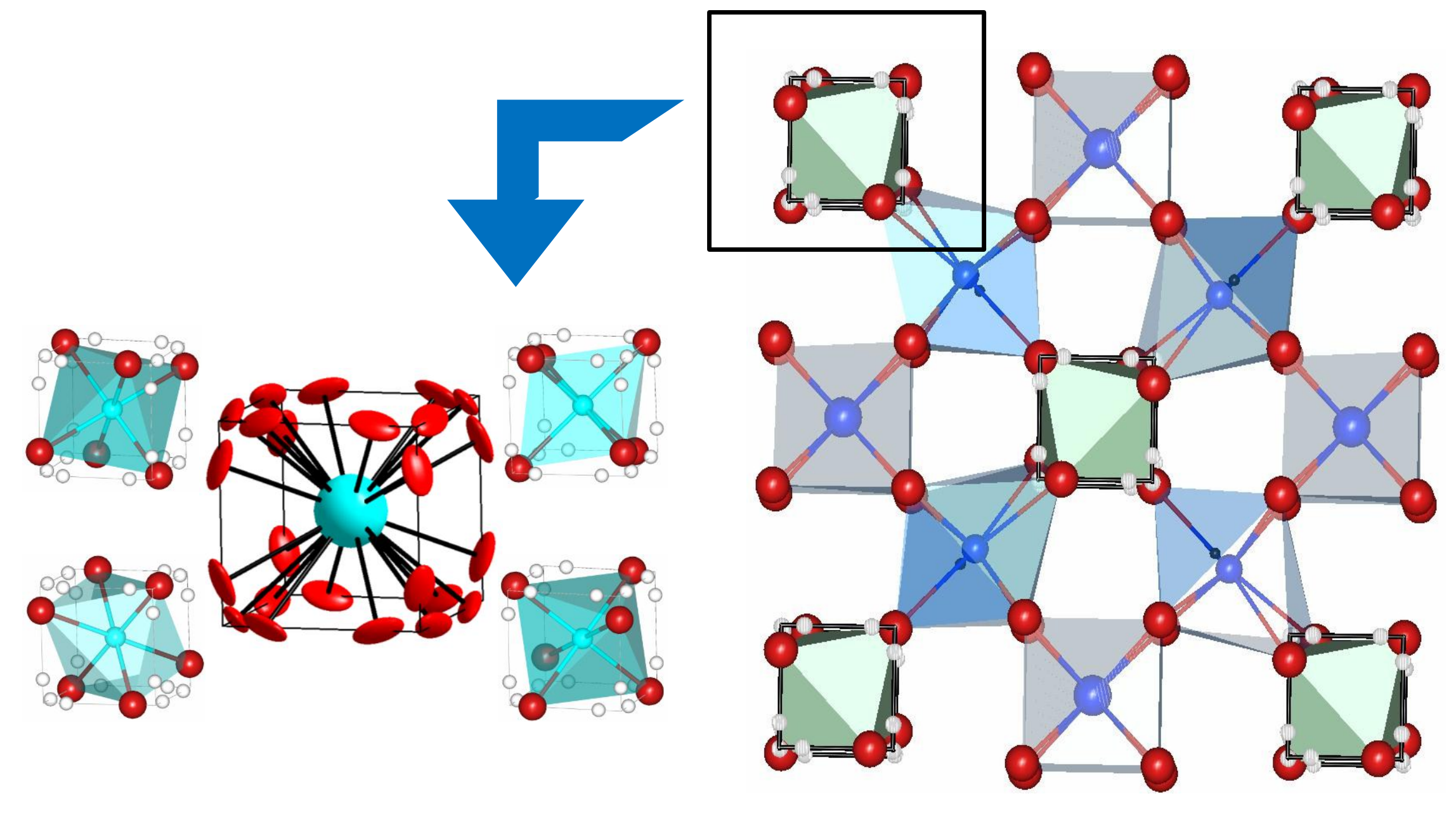

